\title{
Extraction of Pertinent Subsets from Time-Frequency Representations for Detection and Recognition Purposes
}

\author{
Benoit LEPRETTRE* \& Nadine MARTIN \\ LIS \\ Signals and Images Laboratory \\ BP 46, 38402 Saint Martin d'Hères Cedex France \\ nadine.martin@inpg.fr
}

\begin{abstract}
A time-frequency representation can highlight non-stationarities in a signal. We propose to extract subsets from the Time-Frequency Representation (TFR) for classification or recognition purposes. We developed two approaches. The first one is developed for TFRs obtained from the Short Time Fourier Transform or the gliding Minimum Variance method. The extraction of compact subsets is viewed as a segmentation of the TFR, which is performed by morphological filtering and Watershed segmentation. The second approach is developed when the TFR has been obtained using parametric estimators. We consider a hybrid estimator, the ARCAP method, and use a Kalman filter trajectory tracker to extract spectral lines. The proposed methods are illustrated by examples on natural signals : dolphin whistle acoustical signals, cavitation signals and seismic signals produced by snow avalanches.
\end{abstract}

Now with Schneider Electric S.A., Corporate R\&D, Algorithms \& Signal Processing Dept., A2 Research Center, 4, rue Volta, 38050 Grenoble, France - Benoit_Leprettre@mail.schneider.fr

Keywords: Non stationary signal interpretation, Time-frequency representation, Watershed segmentation, Kalman filter, Trajectory tracking, Capon filter, Minimum variance estimator, Autoregressive model, Spectral analysis. 

Résumé

Une représentation temps-fréquence peut mettre en évidence les non-stationarités présentes dans un signal. Nous proposons d'extraire des sous-ensembles ou motifs d'une représentation temps-fréquence (RTF), ceci pour des objectifs de classification ou d'interprétation. Nous avons développé deux approches. La première s'applique sur des RTF obtenus soit par une transformée de Fourier à court terme, soit par la méthode de Minimum de variance glissante. L'extraction de motifs est vue comme une segmentation de la RTF, segmentation réalisée par des filtres morphologiques et par la méthode de la ligne de partage des eaux. La deuxième approche est développée sur des TFR obtenus à partir d'estimateurs spectraux paramétriques. Nous avons considéré un estimateur hybride, la méthode ARCAP, et développé des filtres de Kalman pour réaliser un suivi de trajectoires dans le plan temps-fréquence afin d'extraire les lignes spectrales au cours du temps. Les deux méthodes proposées sont illustrées à l'aide d'applications sur des signaux réels : signaux acoustiques de sifflements de dauphins, signaux de cavitation et signaux sismiques créés par des avalanches de neige. 


\section{Introduction}

Time-frequency (TF) analysis is of great interest when one needs to process a recorded signal in order to make a decision by detecting specific features. Time-Frequency Representations (TFRs) can highlight differences between several types of non-stationary signals and help greatly in the classification or decision task. As a TFR is highly redundant, taking into account a limited number of relevant subsets can improve the quality of the decision. In this paper, we present two approaches for extracting subsets from specific TFRs. The first one, in section 2, is developed for TFRs represented as a full [time, frequency, power or energy] matrix. The second one, in section 3, is developed for TFRs in which only some points are associated to a power or energy value. The proposed feature extraction methods are illustrated with examples on natural signals.

As the purpose of this paper is not to review the different existing TRFs, we just indicate the methods we have used. In the first proposed approach, we use the Gliding Minimum Variance (or Capon's) method assuming a local stationarity [Martin 95], [Durnerin 00]. The structure of the TFR is similar to that of a spectrogram, in the sense that a power value is assigned to each point of the defined TF plane. Figure 1 is an example of such TFR and will be described in section 2.2. However, the achieved TF resolution is higher and the variance of the estimator is lower. The obtained TFR is a full $N_{w} \mathrm{x} N_{f}$ matrix $\underline{\underline{P}}$, where element $P[n, f]$ is the power of the signal at discrete time $n$ and at frequency $f . N_{w}$ (resp. $N_{f}$ ) is the number of points in time (resp. frequency). The aim here is the extraction of compact TF patterns. This first method is well suited to process narrow and broad band signals.

In the second approach, we use the gliding ARCAP estimator [Martin 95], [Padovèse 96], [Leprettre 98]. This hybrid method estimates the signal frequencies from an AR modeling of the signal. At these estimated frequencies, the power values are estimated by Capon's estimator. Unlike the Minimum Variance TFR or the spectrogram, the obtained ARCAP TFR, a $N_{w} \mathrm{x} N_{f}$ matrix $\underline{\underline{P}}$ too, is not of full-matrix type. It is a "cloud of points"type TFR. With a model of order $M_{A R}$, only $N_{f}=M_{A R} / 2$ frequency points with corresponding powers are identified at each time $n$ instead of a complete spectrum. By juxtaposing this set of points on a 3D time-frequency-power 
coordinates system, separated, coherent spectral lines appear. Figure 4-a is an example of such TFR and will be described in section 3.2. If $M_{A R}$ was set correctly, these spectral lines represent the somehow organized TF components of the signal. We call TF trajectories those relevant spectral lines that we aim to extract. As it highlights spectral lines, the ARCAP TF estimator is suited to process narrow-band signals or signals made of several pure frequencies.

\section{Extraction of Subsets from the Matrix-Type TFRs}

As the obtained TFR is a $\mathrm{N}_{\mathrm{w}} \mathrm{xN}$ f matrix, the extraction of a subset can be seen as an image segmentation problem. Several techniques have been developed in the fields of Image Processing and Pattern Recognition. However, a TFR is a very particular type of image. The generation process of the image is known: it consists of successive estimated spectra juxtaposed along the time axis. The method and the parameters (e.g. window length, overlap) used for the analysis are known. A priori knowledge is available: the characteristics of the analyzed signal may allow selecting the areas of interest inside which patterns should be sought for. A TFR is anisotropic: the dimensions along the axes of the image are physically different (time, frequency, power). It has a relevant amplitude structure: in some cases, one needs to extract patterns that are not iso-energetic. Patterns corresponding to transient, evanescent signals produced by sources of varying power (e.g. animals sounds) may not be completely extracted with an iso-energetic method. In that case, nonlinear filters can be an interesting alternative. We propose to apply morphological operators.

\subsection{Extracting features from the TFR using Mathematical Morphology}

We propose to use methods based on Mathematical Morphology to take the a priori knowledge into account and extract the pertinent subsets [Pierson 97]. Two steps corresponding to Beucher's segmentation paradigm [Beucher 90] can be considered in the proposed approach.

Step 1: Modeling the a priori knowledge by transforming the TFR 
Let $\underline{P}=P[n, f]$ be the considered TFR matrix obtained using Minimum Variance estimation. The procedure is the following: 1) Define criteria that allow distinguishing between the noise and the interesting patterns, e.g. the peak power of a pattern and / or the contrast between a peak of the TFR and the surrounding background noise. 2) According to the selected criteria, define and calculate a transform of the TFR that highlights the frontiers between the patterns of interest and the background noise. This transform changes the original TFR into $P^{\prime}[n, f]$ that will eventually be segmented. If the criterion is the contrast, the corresponding transform can for example be the modulus of the morphological gradient defined in [Serra 82]. 3) Use this function to mark in $P^{\prime}[n, f]$ the areas of interest (patterns to extract) and these corresponding to the noise. The markers can be obtained by simply thresholding $P^{\prime}[n, f]$ or using more elaborate morphological transforms (e.g. a combination of Top-Hat Transforms, erosions, dilations, etc.), depending on the characteristics of the TFR and on the complexity of the recognition problem. This part of the procedure, sometimes called the "intelligent" part, involves all the user's knowledge in order to try understanding the TFR.

\section{Step 2: Extracting subsets automatically using the Watershed method}

The second step of the procedure is automatic and is aiming to really extract the relevant subsets. First, a modified function $P^{\prime \prime}[n, f]$ is reconstructed by eroding the noise markers. Then, the Watershed method [Beucher 90][Vincent 91] is used on $P^{\prime \prime}[n, f]$ in order to obtain closed contours following the local maxima of $P^{\prime \prime}[n, f]$, i.e. the ridgelines of the TFR. The interesting point is that this method is non parametric and the obtained subsets are not necessarily iso-energetic. The Watershed method thus allows to extract contours with non homogenous power, or to extract interesting patterns of different power levels from a TFR.

\subsection{Application on real data}

The signal on figure 1 is a dolphin communication whistle recorded using a $32 \mathrm{kHz}$ sampling frequency. Capon's RTF (figure 1a) shows that the interesting features are successive transient signals modulated in both amplitude and frequency. Energetic patterns above $13 \mathrm{kHz}$ are eliminated due to anti-aliasing filtering. A highly-energetic, colored noise is present. A priori knowledge on that signal allows the definition of two criterions. The first one is 6 
based on the amplitude. The high amplitude peaks $[31.5 \mathrm{~dB}, 50.5 \mathrm{~dB}]$ are the interesting signatures. Few of them arise from the background noise $[20 \mathrm{~dB}, 31.5 \mathrm{~dB}]$. The second criterion characterizes the mean frequency bandwidth of the peaks and is obtained after several adapted Top-Hat transforms applied with a vertical linear structuring element [Pierson 97]. It is in fact a size criterion. For that signal, we obtain a mean bandwitdh of 1570 Hz. Signal and noise markers are calculated from this knowledge (figures 1b-c). Details of the procedure are in [Pierson 97]. After this first step, which must be adapted to each context, the watershed method is applied. Figure $1 \mathrm{~d}$ shows that only the features of interest are extracted. Classical iso-energetic segmentation (figure 1e) extracts many noise contours in addition to the interesting features. The counter-part of good performance of our approach is that it cannot be applied without a priori knowledge of the signal. But it yields significant results for both frequency and amplitude modulated signals in colored noise. Another difficult example, a cavitation signal, is presented in figure 2. The watershed segmentation yields results closer to the interpretation of human experts (the "golden ears").

\subsection{Interpreting the $T-F 2 D$ contours}

The original TFR has just been reduced to a limited number of closed contours defining its most pertinent subsets. Several parameters can directly be derived from these contours, for example: position (time and frequency) of the center of gravity of the subset, duration (i.e. extension along the time axis), bandwidth (i.e. extension along the frequency axis), average power of the time-frequency points inside the contour, orientation with respect to the axes, etc. A review of several classical methods for describing contours can be found in [Marinovic 85] and several applications in [Granlund 72], [Chassery 91]. The description parameters can in turn be used as the input of a decision system based e.g. on expert reasoning or statistical analysis, depending on whether an exhaustive training set of signals is available or not. Interpration of TFR's after a morphological non linear filtering can be found in [Pierson 97]. 
Figure 1 Dolphin Communication whistles in a submarine acoustic

a- Capon TFR, $N f=256, M=20, L=127(4 \mathrm{~ms}), \delta L=64(2 \mathrm{~ms}), F e=32 \mathrm{KHz}$

b. Signal markers

c. Noise markers

d. Result of the watershed

e. Result of isolevel segmentation at $31.5 \mathrm{~dB}$ from the minimal power
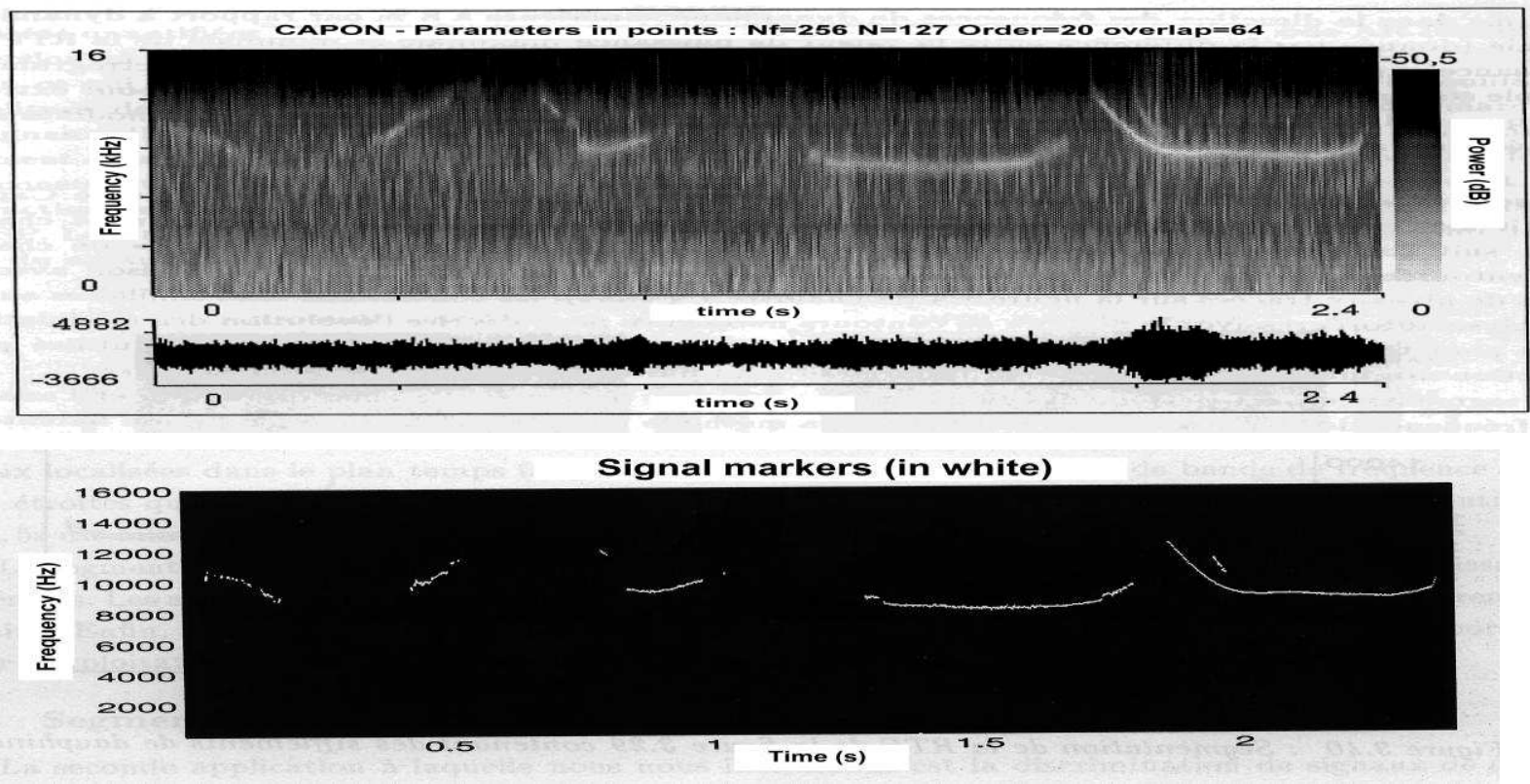

(c) Noise markers (in white)

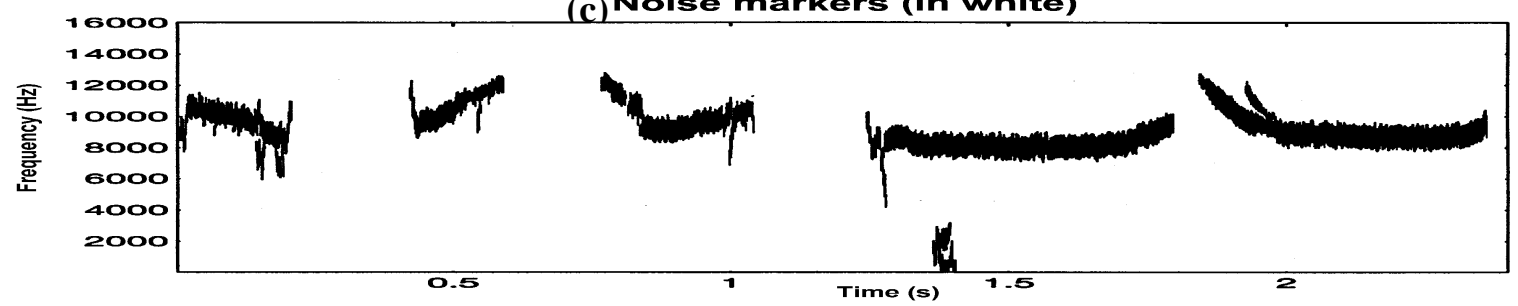

(d) Result of the Watershed algorithm

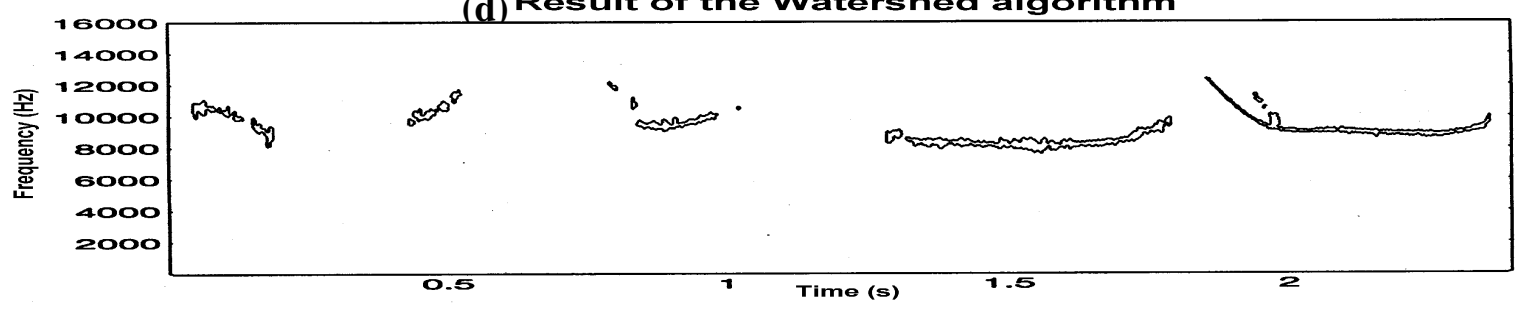

(e)Isolevel segmentation (31.5dB from minimal power)

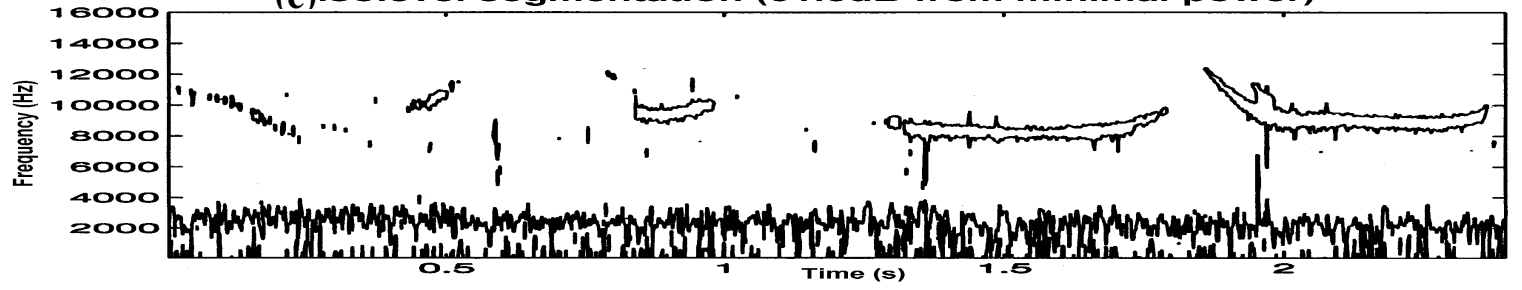




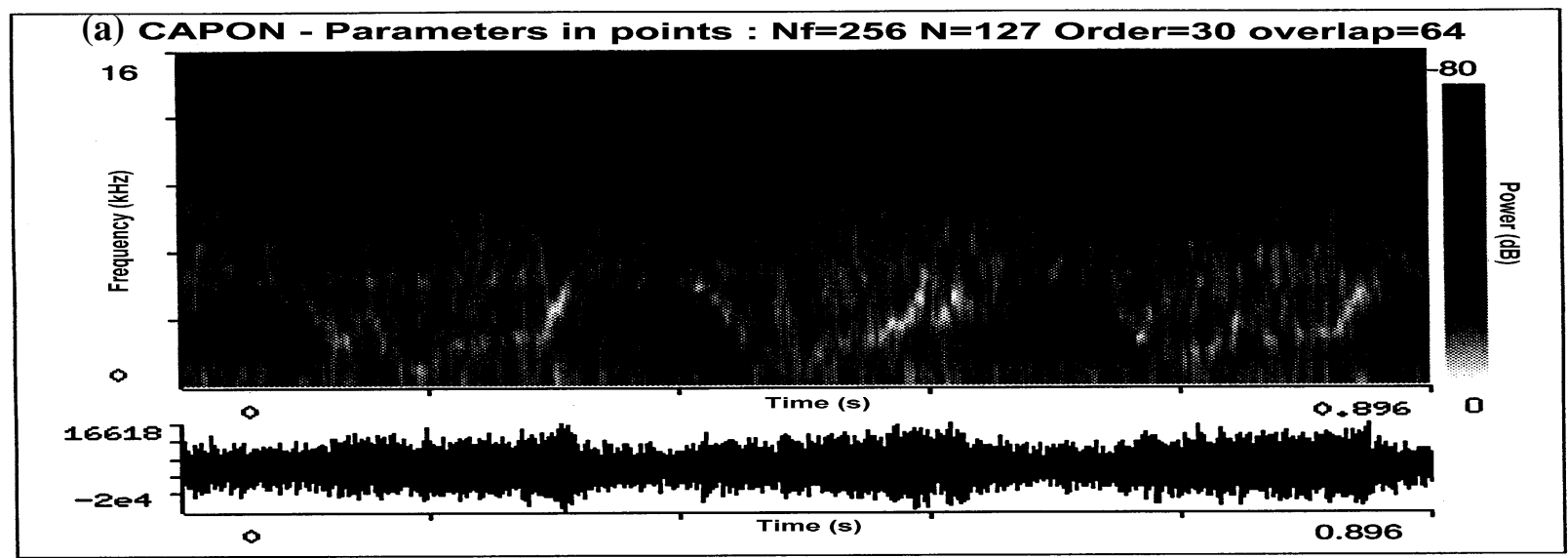

(b) Result of the Watershed algorithm

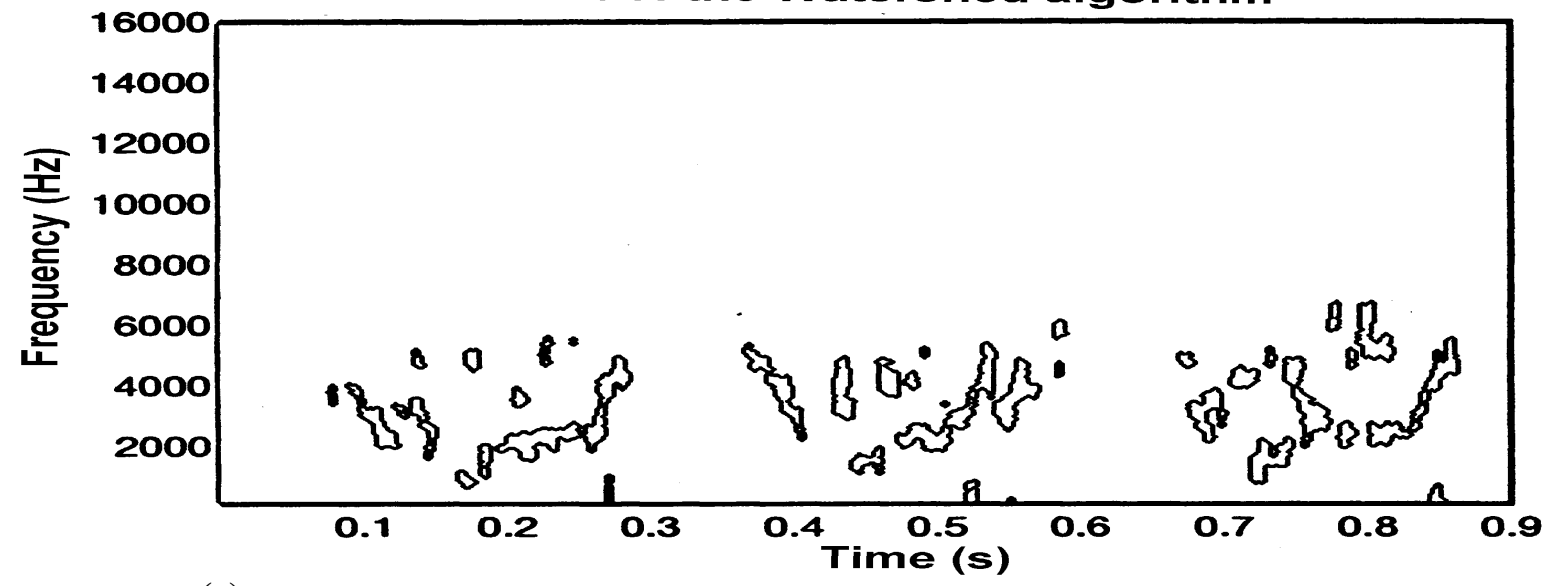

(c) Isolevel segmentation (-10dB from maximum power)

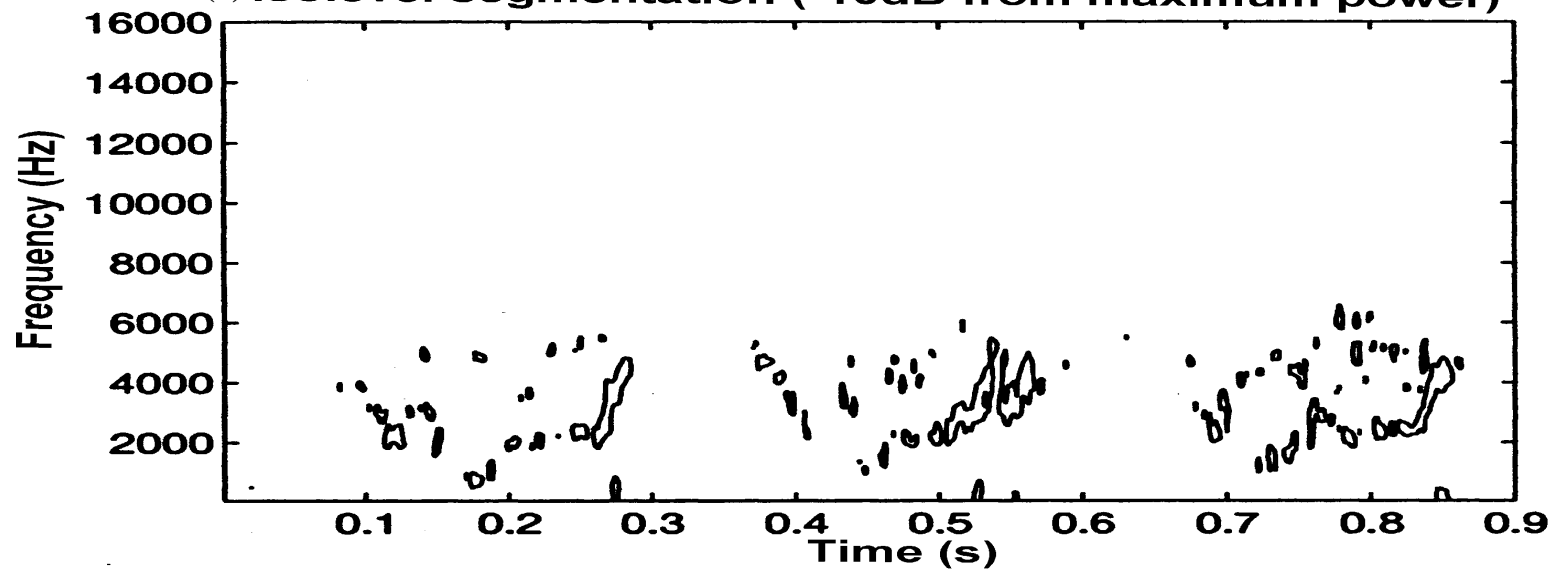

Figure 2 Cavitation signal

a. Capon TFR, $N f=256, M=30, L=127(4 \mathrm{~ms}), \delta L=64(2 \mathrm{~ms}), F e=32 \mathrm{KHz}$

b. Result of the watershed

c. Result of isolevel segmentation at $-10 \mathrm{~dB}$ from the maximal power 


\subsection{Limits of the gradient method for segmenting TFRs}

Lots of segmentation technics are based on a gradient function. It may be of some interest to study not the influence of the noise, which is known to be significant, but the influence of the signal spectral bandwidth on the gradient function. Without any loss of generality, let us consider the estimate $\hat{\gamma}_{x}(v)=|X(v)|^{2}$ of the periodogram of a stochastic signal $x(t)=s(t)+b(t)$ where $X(v)$ is its Fourier Transform, $s(t)$ a deterministic signal with spectrum $\gamma_{s}(v)$ and $b(t)$ a gaussian white noise with variance $\sigma^{2}$.

$\hat{\gamma}_{x}(v)$ is a noncentral chi-square random variable whose mean is $E\left\{\hat{\gamma}_{x}(v)\right\}=\sigma^{2}+\gamma_{s}(v)$ and variance $\operatorname{Var}\left\{\hat{\gamma}_{x}(v)\right\}=\sigma^{4}+2 \sigma^{2} \gamma_{s}(v)$. Therefore, the gradient function in the frequency direction $\hat{G}(v)=\frac{\partial \hat{\gamma}_{x}(v)}{\partial v}$ is a random variable whose mean is the derivative of $E\left\{\hat{\gamma}_{x}(v)\right\}$ and second order moment is $E\left\{\hat{G}(v)^{2}\right\}=-\left(\frac{\partial^{2} \Gamma_{\gamma}(\eta)}{\partial \eta^{2}}\right)_{\eta=0}$ with $\Gamma_{\gamma}(\eta)=E\left\{\hat{\gamma}_{x}(v) \hat{\gamma}_{x}(v-\eta)\right\}$. From these relations, we can deduce the variance of $\hat{G}(v):$

$\operatorname{Var}\{\hat{G}(v)\}=\left(\gamma_{s}(v)+\sigma^{2}\right)\left(-\frac{\partial^{2} \gamma_{s}(v-\eta)}{\partial \eta^{2}}\right)_{\eta=0}$

Let us consider $s(t)$ as a gaussian envelope, $s(t)=A \cdot e^{-4 \pi^{2} \Delta v^{2} t^{2}}$ with amplitude $A$ and spectral width $\Delta v$.

Setting $A=2 \sqrt{\pi} \Delta v$ for having $A$ independent of $\Delta v$, we get $\gamma_{s}(v)=e^{-v^{2} / 2 \Delta v^{2}}$. Inserting into (1), we obtain

$$
E\{\hat{G}(v)\}=\frac{|v|}{\Delta v^{2}} e^{-v^{2} / 2 \Delta v^{2}} \quad \text { and } \quad \operatorname{Var}\{\hat{G}(v)\}=\frac{1}{\Delta v^{2}} e^{-v^{2} / \Delta v^{2}}+\frac{\sigma^{2}}{\Delta v^{2}} e^{-v^{2} / 2 \Delta v^{2}}\left(1+\frac{v^{2}}{\Delta v^{2}}\right)
$$

The mean maximum is reached for $v= \pm \Delta v$. At this frequency, the mean and the variance of the gradient function are 
$E\{\hat{G}(\Delta v)\}=\frac{1}{\Delta v} e^{-1 / 2} \quad$ and $\quad \operatorname{Var}\{\hat{G}(\Delta v)\}=E\{\hat{G}(\Delta v)\}^{2}\left(1+2 \sigma^{2} e^{1 / 2}\right)$

As a major result, when the spectral bandwidth $\Delta v$ increases, the mean maximum varies with $1 / \Delta v$ and so is rapidly lowering. Furthermore, the standard deviation, square root of the variance, is greater than the mean even for high signal to noise ratio. Figure 3-a shows results for four values of $\Delta v$ without additive noise whereas figure 3-b shows the fast increase of the variance even for a signal to noise ratio of only $30 \mathrm{~dB}$.
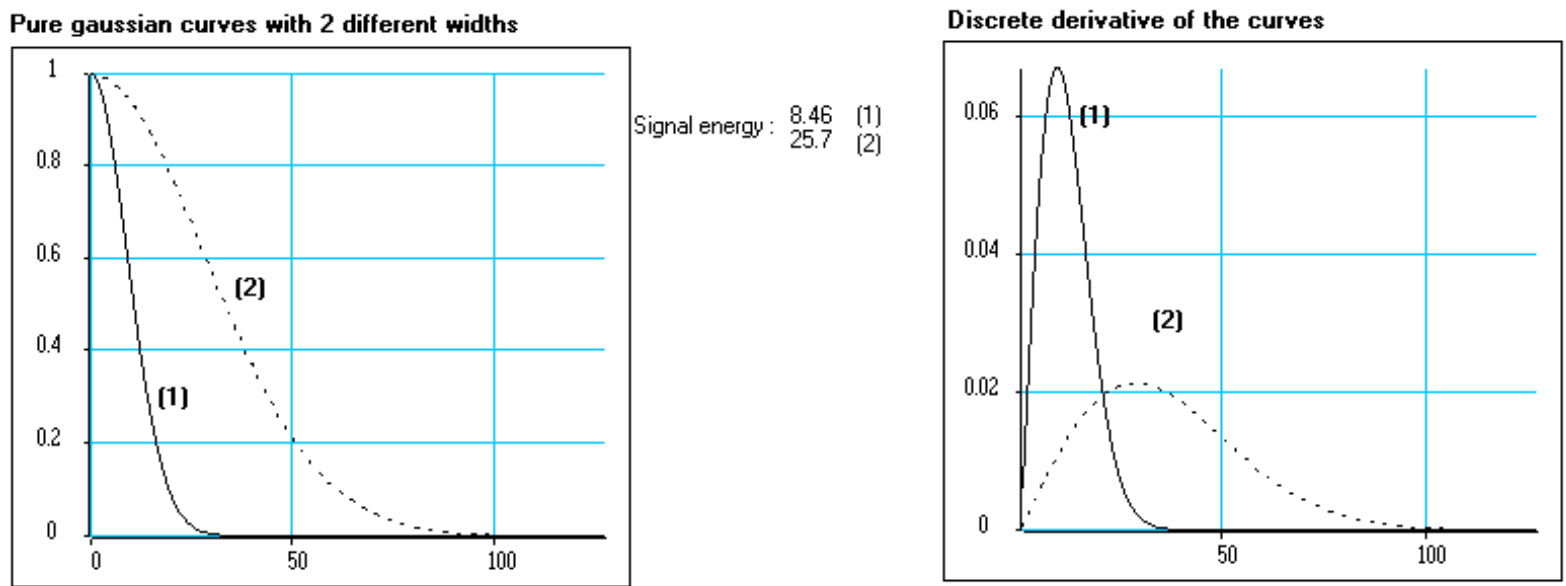

a. without noise
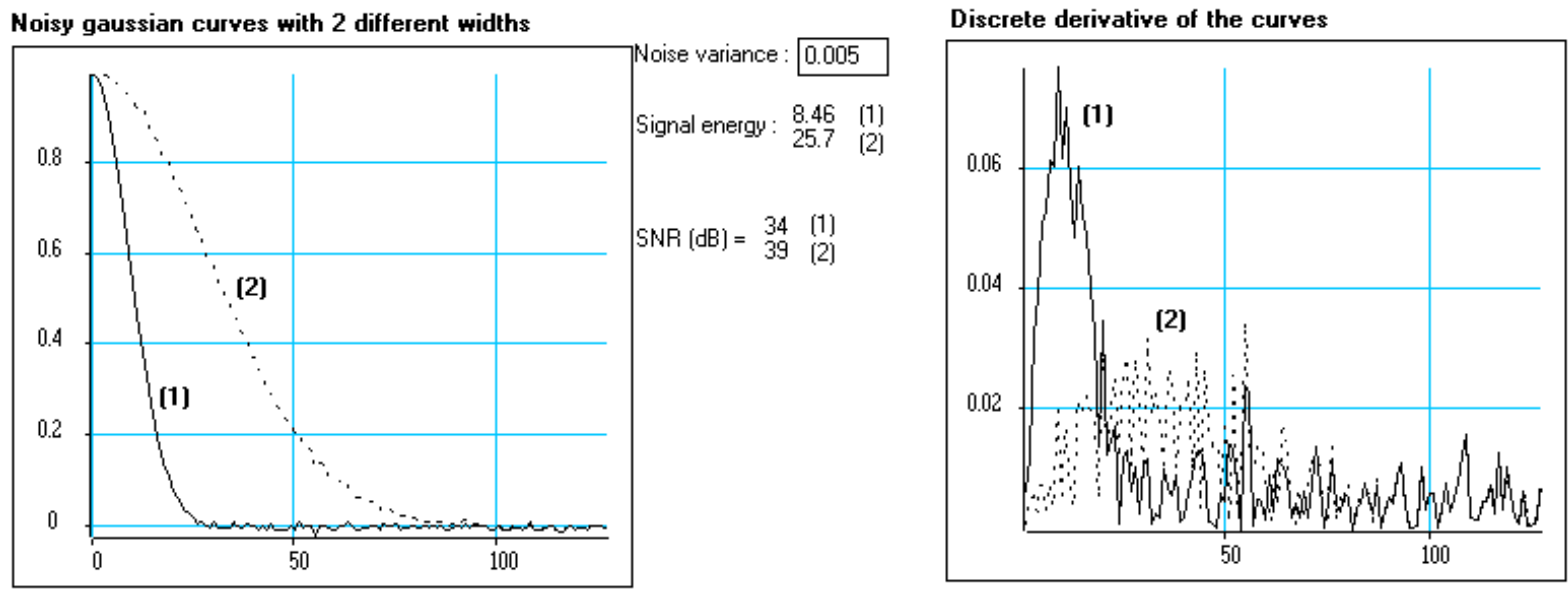

b. with additive noise

Figure $3 \quad$ Spectrum of a Gaussian envelope and its discrete derivative for 2 different widths 
The variation of the gradient with $1 / \Delta v$ creates a too strong dependence of this function with the spectral bandwidth of the signal even for high signal to noise ratios. So, the gradient function fails in highlighting wide band patterns. This property considerably limits the use of this type of segmentation for real signals.

\section{Extraction of Subsets from TFRs of Cloud-of-points Type}

In this paragraph we consider the particular case of an ARCAP TFR, but a similar methodology can be carried out with TFRs obtained by e.g. extracting peak values from a matrix-type TFR.

\subsection{Extracting features from the TFR using Kalman Filtering}

If the signal is made of one or several narrow-band components, some of the points of the ARCAP TFR are structured so that they form coherent lines (so-called time-frequency "trajectories") representing the main TF components of the signal: pure or modulated frequencies. However, in the context of gliding parametric estimation, the link between the estimated parameters at successive dates is lost during the analysis process. What we propose to do is to link the points corresponding to the same frequency component together in order to extract and interpret the identified time-frequency trajectories.

If the SNR is high and the simultaneous frequencies are not too close to each other, a simple thresholding of the ARCAP TFR followed by a nearest neighbor assignment procedure can be used to link the points together and identify the coherent lines [Leprettre 96] [Leprettre 98]. 


\begin{tabular}{|c|c|c|}
\hline Modulation & Model & Particularities \\
\hline $\begin{array}{l}\text { Linear } \\
v_{i}(t)=\alpha \cdot t+v_{0}\end{array}$ & $\begin{array}{l}\text { Ballistic model of order } 1 \\
\qquad\left\{\begin{array}{l}v_{i}(t+\Delta t)=v_{i}(t)+\mathrm{v}(t) . \Delta t+\varepsilon(t) \\
\mathrm{v}(t+\Delta t)=\mathrm{v}(t)\end{array}\right.\end{array}$ & $\begin{array}{l}\mathrm{v}(\mathrm{t})=\alpha \text { constant speed } \\
\text { null acceleration }\end{array}$ \\
\hline $\begin{array}{l}\text { Parabolic } \\
v_{i}(t)=\alpha \cdot\left(t-t_{0}\right)^{2}+v_{0}\end{array}$ & \multirow{3}{*}{$\begin{array}{l}\text { Ballistic model of order } 2 \\
\left\{\begin{array}{l}v_{i}(t+\Delta t)=v_{i}(t)+\mathrm{v}(t) \cdot \Delta t+\frac{\gamma(t)}{2} \cdot \Delta t^{2}+\varepsilon(t) \\
\mathrm{v}(t+\Delta t)=\mathrm{v}(t)+\gamma(\mathrm{t}) \cdot \Delta t \\
\gamma(t+\Delta t)=\gamma(t)\end{array}\right.\end{array}$} & $\begin{array}{l}\mathrm{v}(\mathrm{t})=2 \alpha \cdot\left(\mathrm{t}-\mathrm{t}_{0}\right) \\
\gamma(\mathrm{t})=2 \alpha \text { constant acceleration }\end{array}$ \\
\hline $\begin{array}{l}\text { Hyperbolic } \\
v_{i}(t)=\frac{v_{0}}{1+a . t}\end{array}$ & & $\begin{array}{l}\text { After second-order Mac Laurin } \\
\text { development: } \\
\mathrm{v}(\mathrm{t})=\mathrm{a} v_{0} \cdot(2 \mathrm{at}-1) \\
\gamma(\mathrm{t})=2 \mathrm{v}_{0} \cdot \mathrm{a}^{2}\end{array}$ \\
\hline $\begin{array}{l}\text { Sinusoidal } \\
v_{i}(t)=b \cdot \sin (\omega t+\varphi)+v_{0}\end{array}$ & & $\begin{array}{l}\text { After second-order Mac Laurin } \\
\text { development : } \\
\mathrm{v}(\mathrm{t})=\mathrm{b} \omega(\cos \varphi-\omega \cdot \sin \varphi \cdot \mathrm{t}) \\
\gamma(\mathrm{t})=-\mathrm{b} \omega \cdot \sin \varphi\end{array}$ \\
\hline
\end{tabular}

\section{Table $1 \quad$ Frequency modulations and related ballistic models}

If the SNR of the signal is small, the choice of the parameters for finding the neighbor is difficult and thus a more elaborate procedure is needed. We propose to model the frequency modulation law of the searched trajectories. The problem is thus connected to that of following up the trajectory of a ballistic object, which can be solved using Kalman filtering. Table 1 shows four types of frequency modulations with corresponding ballistic models.

We propose to use two separate Kalman filters in order to take both the frequency variations and the power changes into account. So, the state vector is defined by $\underline{X}(n)=[u(n), v(n), g(n)]^{T}$ with $v(n)$ the first derivative (or speed) and $g(n)$ the second derivative (or acceleration) of $u(n)$, which represents either the estimated frequencies or the estimated powers. Thus, from a Taylor expanding of $u(n)$, the state model of order 2 writes as

$$
\left\{\begin{aligned}
\underline{X}(n+1) & =\left[\begin{array}{ccc}
1 & 1 & 1 / 2 \\
0 & 1 & 1 \\
0 & 0 & 1
\end{array}\right] \cdot \underline{X}(n)+\left[\begin{array}{c}
1 / 6 \\
1 / 2 \\
1
\end{array}\right] \cdot \varepsilon(n) \\
y(n) & =\left[\begin{array}{lll}
1 & 0 & 0
\end{array}\right] \underline{X}(n)+e(n)
\end{aligned}\right.
$$


The errors $\varepsilon(n)$ with variance $Q$ and $e(n)$ with variance $R$ are uncorrelated white noises representing the errors on the model and on the observations $y(n)$ respectively. Let be $\underline{X}_{f}(n)$ the state vector of the first filter where the first component is one of the estimated frequencies at time $n: u_{f}(n)=f(n)$, then let $R_{f}$ and $Q_{f}$ be the error variances. Likewise, let $\underline{X}_{P}(n)$ be the state vector of the second filter where $u_{P}(n)=P[n, f]$, the amplitude of the TFR, and so $R_{P}$ and $Q_{P}$. This parallel modeling of frequencies and amplitudes is possible due to the fact that the variables $f$ and $P$ are independent. The algorithms are initialized with one observed point of the ARCAP TFR with null speed and acceleration. For each filter, the vector $\underline{\hat{X}}(n+1 / n)$, prediction of $\underline{X}(n+1)$ given $\underline{X}(n)$, is gained from the observation at time $n$ given $\hat{u}_{f}(n+1 / n)=\hat{f}$ and $\hat{u}_{P}(n+1 / n)=\hat{P}$. Then, in the time-frequency space, at time $n+1$, an observed point is searched for within a given interval defined with the variance $\left(R_{\mathrm{f}}+\right.$ Variance $\left.(f)\right)$ for the frequency and $\left(R_{P}+\operatorname{Variance}(P[n, f])\right)$ for the power. Several possible points may be candidates. The nearest point $\left(n+1, f_{m}, P\left[n+1, f_{m}\right]\right)$ is assigned to the component according to the minimum of Mahalanobis distance $\mathrm{d}_{\mathrm{M}}$ [Chui 87] defined by:

$d_{M}=\frac{\left(f_{m}-\hat{f}\right)^{2}}{\operatorname{Var}\left(f_{m}-\hat{f}\right)}+\frac{\left.\left(P\left[n+1, f_{m}\right]-\hat{P}\right]\right)^{2}}{\operatorname{Var}\left(P\left[n+1, f_{m}\right]-\hat{P}\right)}$

This matched point becomes the observation at time $n+1$ from which estimation $\underline{\hat{X}}(n+1 / n+1)$ is set for each filter and according to the variance parameters $Q_{f}, R_{f}, Q_{P}$ and $R_{P}$. This provides $\hat{u}_{f}(n+1 / n+1)=\hat{\hat{f}}$ and $\hat{x}_{P}(n+1 / n+1)=\hat{\hat{P}}$. If no point is found in the given interval, the current trajectory dies and another trajectory is initiated using a point of the TFR not already assigned to a trajectory.

When this procedure is carried out on the whole TFR, what we get is a set of one-dimensional time-frequency components (the so-called spectral lines). Two types of data are available for each line: a matched trajectory containing only points from the original ARCAP TFR and formed with all $\left(n, f_{m}, P_{m}\left[n, f_{m}\right]\right)$ points and a modeled trajectory formed with the output of Kalman filters, the $(n, \hat{\hat{f}}, \hat{\hat{P}})$ points. If the confidence in the model (defined 
by $Q_{f}$ and $Q_{P}$ ) is much higher than the confidence in the observed values (defined by $R_{f}$ and $R_{P}$ ), the modeled trajectory is smoother than the matched trajectory. Shorter lines which have only a few points, the number of which must be set according to the characteristics of the signal, are considered as noise and eliminated. Kalman filtering of order 1 is often satisfactory. Orders higher than 2 could as well be considered [Davy 98]. But, even if the signal is highly modulated, a $2^{\text {nd }}$ order is sufficient. Recursive implementations of Kalman filtering can be found in the literature [Chui 87]. See [Roguet 96] and [Cottereau 00] for specific studies concerning the tracking of ARCAP time-frequency trajectories.

\subsection{Application on a real signal}

ARCAP trajectory tracking has been applied to the recognition of seismic signals produced by snow avalanches and provided by Meteo-France [Leprettre 96][Leprettre 98]. First-order Kalman filters identified four spectral lines. These are shown superimposed with the ARCAP analysis on figure 4-a and as isolated one-dimensional full-scale curves on figure 4-b. For each extracted time-frequency trajectory a couple of parameters are estimated: extreme frequencies, mean and standard deviation. 
[a] Superimposed ARCAP analysis * Kalman trajectory tracking

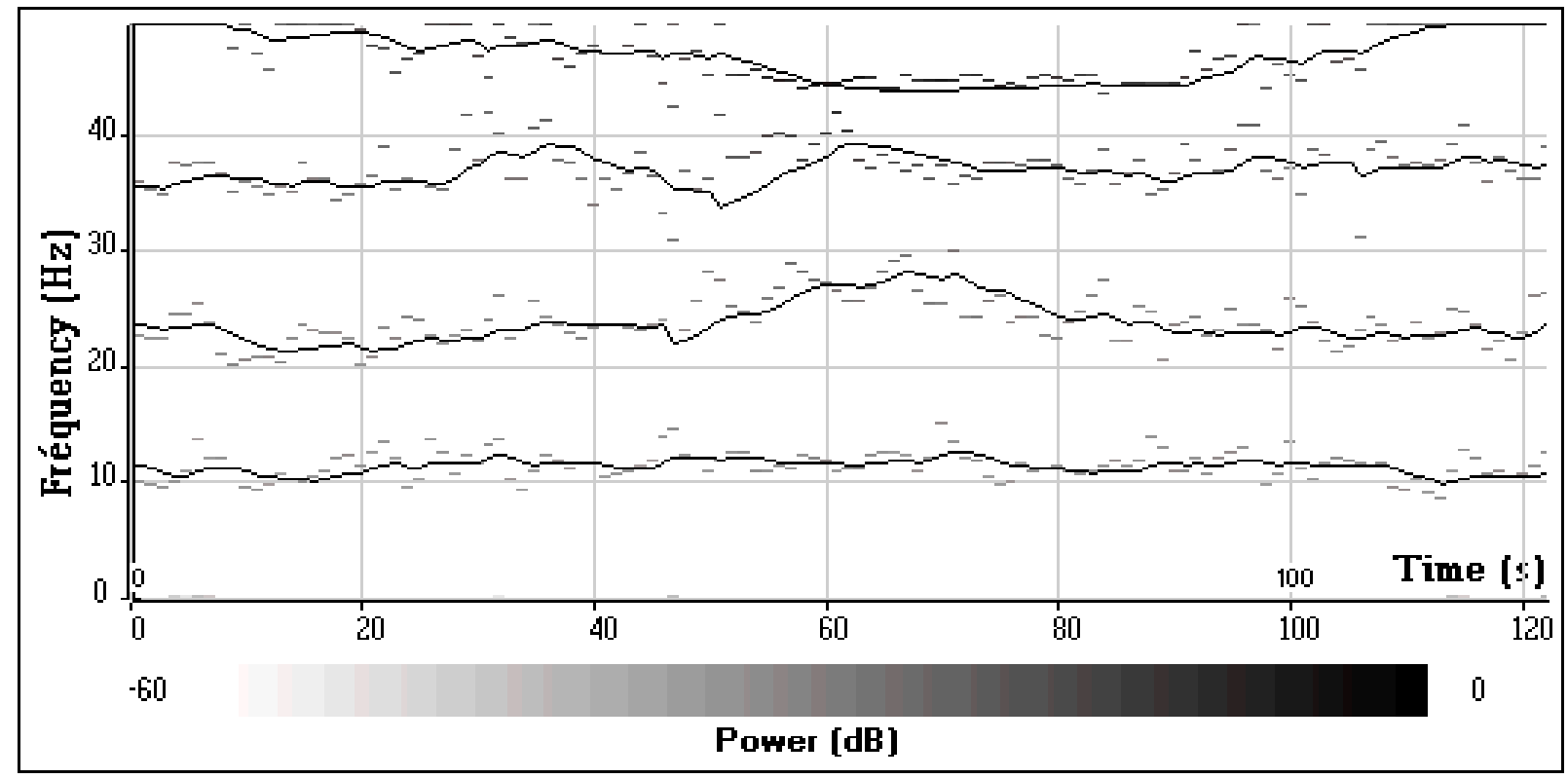

[b] Extracted Kalman trajectories : fréquency and power
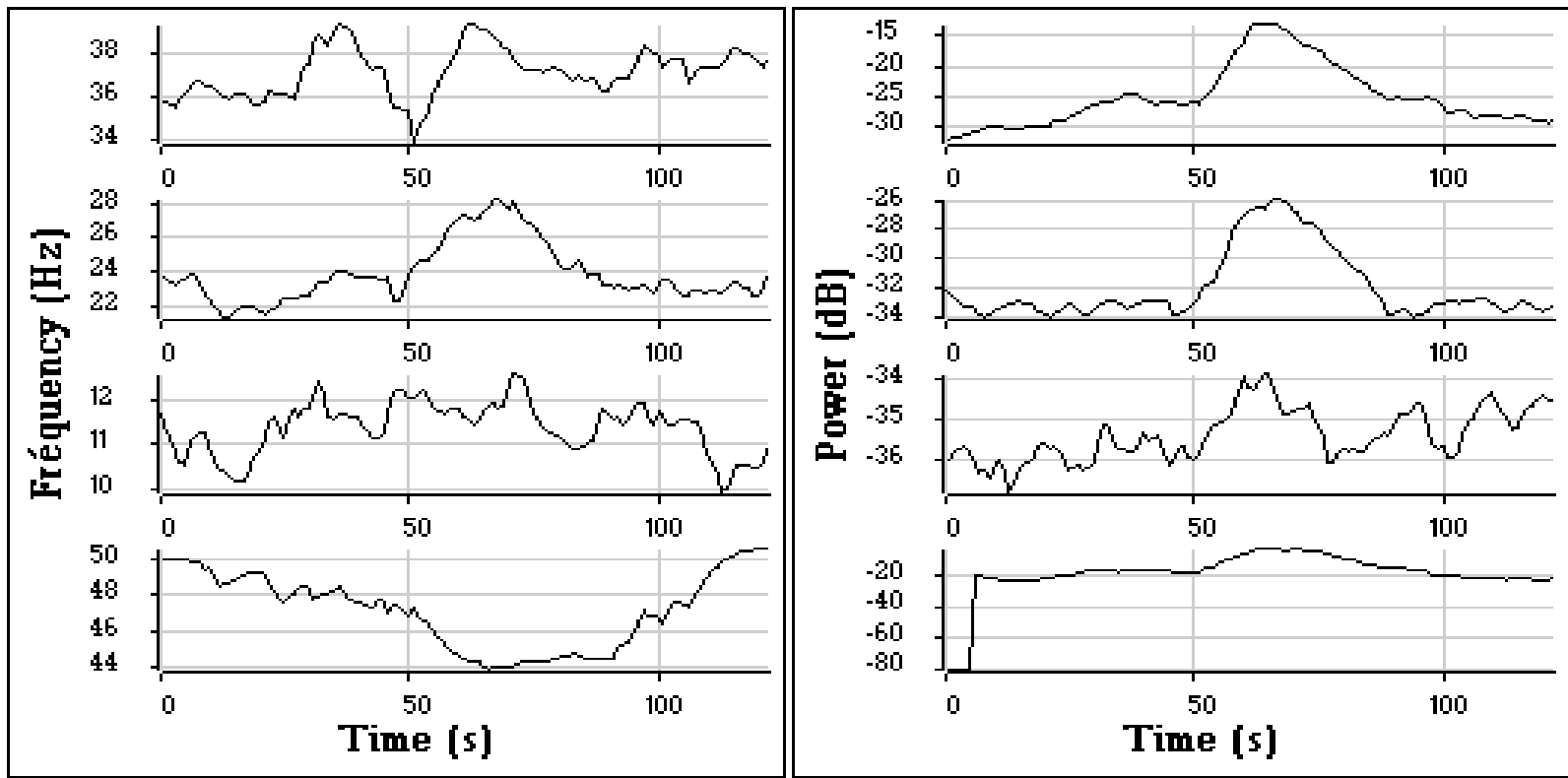

\begin{tabular}{|c|c|c|c|c|}
\hline \multicolumn{5}{|c|}{ Trajectory } \\
\hline \# & Min & $\operatorname{Max}$ & Mean & StDev \\
\hline $\begin{array}{l}1 \\
2 \\
3 \\
4\end{array}$ & $\begin{array}{l}33.95 \\
21.30 \\
9.921 \\
43.99\end{array}$ & $\begin{array}{l}39.45 \\
28.31 \\
12.58 \\
50.49\end{array}$ & $\begin{array}{l}37.15 \\
23.85 \\
11.37 \\
47.16\end{array}$ & $\begin{array}{l}1.119 \\
1.735 \\
0.585 \\
2.069\end{array}$ \\
\hline
\end{tabular}

\begin{tabular}{|c|c|c|c|c|}
\hline \multicolumn{5}{|c|}{ Trajectory } \\
\hline$\#$ & Min & $\operatorname{Max}$ & Mean & StDev \\
\hline $\begin{array}{l}1 \\
2 \\
3 \\
4\end{array}$ & $\begin{array}{l}-32.4 \\
-34.1 \\
-36.8 \\
-80\end{array}$ & $\begin{array}{l}-12.9 \\
-25.8 \\
-33.9 \\
-2.95\end{array}$ & $\begin{array}{l}-25.1 \\
-32.0 \\
-35.4 \\
-19.1\end{array}$ & $\begin{array}{l}5.011 \\
2.397 \\
0.653 \\
15.12\end{array}$ \\
\hline
\end{tabular}

Figure $4 \quad$ Seismic signals produced by snow avalanches a. Superimposed ARCAP analysis and Kalman trajectory tracking b. Extracted Kalman trajectories: frequency and power 


\subsection{Interpreting the $1 D$ spectral lines}

The original ARCAP time-frequency representation is reduced to a limited number of matched and/or modeled spectral lines representing only the most pertinent information of the original TFR. As the extracted components are one-dimensional curves, they are easier to handle than the whole TFR. Several parameters with physical significance can be derived from each component: onset moment, duration, mean frequency and power values, etc. Global trends can be evaluated using e.g. interpolation and lowpass filtering. Ruptures inside a component can be detected using e.g. wavelet analysis or prediction models if needed. The trajectories can also be compared to various models (polynomials, spline functions, etc.) derived from physical considerations or to a library of typical features of interest. The parameters derived from the extracted spectral lines sum up the time-frequency characteristics of the signal in a convenient way. They can be used as the input vector of a decision system for classification purposes. An illustration of this can be found in [Leprettre 98] for the avalanche signal recognition of the previous section.

\section{Conclusion}

Two methods for extracting pertinent information from TFRs have been presented. The first one is based on morphological segmentation and is suitable to matrix-type TFRs like. The second one implements a trajectory tracking Kalman filter and is suitable to TFRs of cloud-of-points type obtained after a modeling process such as ARCAP analysis, or after peak extraction from a matrix-type TFR. Examples on real signals have been presented and commented. Morphological segmentation can produce interesting extraction performance even on complex signals (low signal to noise ratio, colored noise, severe non stationarities in time or in frequency). As a priori knowledge is necessary, it is difficult to propose a global strategy for choosing the required parameters. Every application must be well controlled. The tracking of time-frequency trajectories, only suitable for narrow-band signals, provides a good performance with a low complexity. 
Time-frequency decision is currently fast growing. [Martin 98] reflects the state of the art in the French community. In order to improve the first presented method, we would like to eliminate the drawback produced by the gradient function. A work is in progress with a completely different approach, whose preliminary results are in [Hory 00], [Hory 01].

\section{References}

Beucher S., Segmentation d'Images et Morphologie Mathématique, Ph-D Thesis, ENSMP, Fontainebleau, France, June 1990.

Chassery J.M., A. Montanvert, Géométrie Discrète en Analyse d'Images, HERMES, Paris, 1991.

Chui C.K., G. Chen, Kalman filtering with real-time applications, Springer-Verlag, 1987.

Cottereau H., M. Davy, C. Doncarli, N. Martin, Using ARCAP Time-Frequency Representations for Decision, EUSIPCO, Tampere, Finland, 5 - 8 September, 2000.

Davy M., B. Leprettre C. Doncarli, N. Martin, Tracking of Spectral Lines in an ARCAP Time-Frequency Representation, EUSIPCO, Rhodes, Greek, September 8-11, 1998, pp. 633-636.

Durnerin M., N. Martin, Minimum Variance Filters and Mixed Spectrum Estimation, Signal Processing, Vol. 80, $\mathrm{n}^{\circ}$ 12, December 2000, pp. 2597-2608.

Granlund G.H., Fourier Processing for Hand Print Character Recognition. IEEE Trans. On Computers, Vol. C-21, February 1972, pp. 195-201.

Hory C., N. Martin, A. Chehikian, L.E. Solberg, Time-Frequency Space Characterization Based on Statistical Criterions. EUSIPCO, Tampere, Finland, September 5 - 8, 2000.

Hory C., N. Martin, A. Chehikian, Spectrogram segmentation by Means of Statistical Features for Non-Stationary Signal Interpretation. Submitted to IEEE Signal Processing, July 2001.

Leprettre B., Reconnaissance de signaux sismiques d'avalanches par fusion de données estimées dans les domaines temps, temps-fréquence et polarisation, Ph-D Thesis, University of Grenoble, Oct. 1996.

Leprettre B., N. Martin, F. Glangeaud, J.-P. Navarre, Three-Component Signal Recognition Using Time, TimeFrequency and Polarization Information - Application to Seismic detection of Avalanches. IEEE Trans. On Signal Processing, Vol. 46, No. 1, pp. 83-102, January 1998.

Martin N., J. Mars, J. Martin, C. Chorier, A Capon's Time-Octave Representation. Application in Room Acoustics. IEEE Trans. on Signal Processing,Vol.43, No. 8, pp. 1842-1854, August 1995.

Martin N., Doncarli C., Davy M., Decision Methods in Non Stationnary Signals Context, $\underline{3^{\text {rd }} \text { International }}$ Conference on Acoustical and Vibratory Surveillance, Senlis, France, October 1998, pp.47 - 56.

Marinovic, N.M., G. Eichmann, Feature Extraction and Pattern Classification in Space - Spatial Frequency Domain. SPIE Intelligent Robots and Computer Vision, Vol. 579, 1985.

Padovese L., N. Martin, J.M. Terriez, Méthode Temps-Fréquence Hybride ARCAP pour l'Identification des Caractéristiques Dynamiques d'un Pylône Compression d'un Téléphérique Monocâble, Traitement du Signal, Vol.13, No. 3, Septembre 1996. 
Pierson V., Extraction de sous-ensembles temps-fréquence en vue d'une prise de décision en non stationnaire Application en acoustique sous-marine, Ph-D Thesis, National Polytechnic Institute of Grenoble, April 1997.

Roguet W., N. Martin, A. Chehikian, Tracking of Frequency in a Time-Frequency Representation, IEEE Int. Symp. On TFTS, Paris, France, 1996, pp. 341-344.

Serra J., Image Analysis and Mathematical Morphology. Academic Press, 1982.

Vincent L., P. Soille, Watersheds in Digital Spaces : an Efficient Algorithm based on Immersion Simulations. IEEE Trans. On Pattern Analysis and Machine Intelligence, Vol. 13, No. 6, June 1991; pp. 583-598. 